\title{
Defining solution spaces for customizations
}

\author{
Nikolas Käkelä [0000-0003-0825-4497] and Joakim Wikner ${ }^{2}$ [0000-0003-2252-5337] \\ 1,2 Jönköping University, 551 11, Jönköping, Sweden \\ ${ }^{1}$ nikolas.kakela@ju.se
}

\begin{abstract}
Customization in different flavors have been identified as an important differentiator if low-cost competitiveness is not viable. To provide a customer unique solution is however not the same as providing a solution that is designed and individualized for a particular delivery to a customer. These two cases are illustrations of how customer requirements may be fulfilled differently depending on the match between stated requirements and the solution offered. The range of solutions that can be offered is represented by a solution space consisting of either predefined or postdefined solutions. Predefined refers to solutions that are defined before commitment to a customer and postdefined refers to solutions that are defined after commitment to a customer. Both cases are constrained by a boundary of possible solutions but the postdefined solutions provide opportunities for bounded innovation beyond what the predefined solutions can provide. Combining the properties of the different solution spaces provides not only an operational definition of customization but also supports in identifying strategic opportunities for extending the solutions and types of customizations a business provides.
\end{abstract}

Keywords: Customization, solution space, decoupling point.

\section{Introduction}

Increased proliferation of demand has led to suppliers' adoption of a variety of customer driven production practices. Several research contributions have been made to provide further understanding of these practices. Hoekstra, Romme [1] suggested logistics structures based on decoupling points to illustrate how customer driven production approaches can be classified. In this context the Configure-to-order (CTO) and Engineer-to-order (ETO) systems diverge from other production approaches by being comprised of customization activities that are driven by commitment to a customer rather than driven by speculation.

Within the CTO-approach, the possible range of customized solutions is designed or engineered before commitment to a specific customer, but then configured in accordance to each customer's needs once the customer order is received. Notably, the customized solution is generated within the boundaries of the predefined configuration possibilities. In this sense it differs from the ETO-approach. For ETO the engineering activities are performed in line with each customer's needs and there are in most cases 
no predefined solutions or alternatives to select from when customizing. This should however not be interpreted as that the customers freedom of choice is without constraints. ETO-companies have boundaries as to what orders they embark on too, but the boundaries are operationalized differently.

In this research, the set of possible solutions when customizing is referred to as the solution space that represent what can be offered by the supplier. The solution space is decisive for the range of solutions that can be offered to the customer. Some suppliers establish clear boundaries in advance as to what solutions they offer, allowing production processes to be stable by decreasing product variety. Others benefit from not having the solution space constrained and instead provide products fully tailored to customers' needs. Regardless of the position of the supplier on this matter, the solution space is both an operational and strategic concern for suppliers offering customized products. The purpose of this research is therefore to define solution spaces that embrace different types of customization.

\section{Methodology}

The conceptual approach that this research is based on is derived from the researchers' experiences from research projects focusing on customization related topics. The research is conducted with an analytical conceptual approach, using logical relationshipbuilding rather than empirical data for theory development [2].

\section{Categories of flow drivers}

Customization is by definition based on customer requirements but the preconditions for customization are established before commitment to a customer. This differentiation is in line with the decoupling of speculation driven activities and commitment driven activities provided by the customer order decoupling point (CODP) [1]. The CODP is a time-based concept that is defined based on the relation between the time the customer must wait for delivery to be performed and the total lead-time required to perform all activities related to the delivery. Activities performed when the customer is waiting is here referred to as commitment driven (CD) activities and activities that must be performed in advance of committing to a customer is here referred to as speculation driven (SD) activities. SD activities are based on expectations about future requirements that may not be perfectly in line with future customer requirements related to $\mathrm{CD}$ activities. To cover for this difference the interface between SD and CD is usually associated with a stock point that decouples the two types of flow: SD and CD. In relation to customization it is obviously not suitable to combine customization with SD since the actual customer requirements are not known at that instant in time. CD activities are however well suited for customization but at the same time it does not exclude the delivery of standardized products to customer order, i.e. CD. Even if customization and CD are intimately connected there are several different types of customizations that may be performed and this is related to the solution space of the customized solutions. 


\section{Categories of solution spaces}

While a mass producer must identify the universal needs shared by its target customers, a mass customizer instead identifies the idiosyncratic needs of their customers. By doing this, the mass customizer can predefine alternative solutions that customers can demand, and engineering activities (note that "engineering" here basically represents all types of activities not related to the physical delivery, i.e. provisioning, of the product) can in this way be SD. However, for customizers, where these engineering activities are $\mathrm{CD}$, the activity of identifying the needs of a customer is repeated for each individual commitment. It becomes clear that there is a fundamental difference in defining solutions where engineering activities are SD rather than being CD.

\subsection{Predefined and postdefined solutions}

To further distinguish SD solutions from CD solutions, the solutions relation to the CODP can be considered. A solution that is SD is in this research referred to as a predefined solution meaning that the customized solution is defined upstream from (before) the CODP. It is a customization system where the possible solutions have been clearly defined before the customer order is received. In contrast, a CD solution is here referred to as a postdefined solution. Consequently, only downstream of (after) the CODP does the solution become clearly defined and the constraints actuated.

For predefined solutions, customers need to keep their adaptations within the confines of a set of established solutions or list of options. It limits the customer's freedom of choice but allows the supplier to establish stable processes for providing the customization, enabling low-cost adjustments to the production process [3].

For postdefined solutions, the customer is less constrained. Here, the customer can get response for truly individualized requirements as there are no predefined solutions that the customer chooses from. However, while customers are free to make inquires without any constraints, the suppliers always have some sort of boundary as to what commitments they take on. The suppliers might for example turn down customer orders that go far beyond their area of expertise.

\subsection{Solution spaces}

Predefined and postdefined solutions can be further defined in terms of solution spaces. The concept of "solution space" is used in for example optimization theory where it refers to the set of all possible points that satisfy a problem's constraints. The concept has also been applied for innovation toolkits and mass customization, referring to the set of possible solutions that a customer can choose from when a supplier provides customizations. This application of the concept does however not portray a comprehensive understanding of the role of solution spaces for customization. To address this, three types of solution spaces can be identified in relation to some key product delivery strategies as illustrated in Fig. 1.

For standardized products or services, the solution space can be considered as "collapsed" into one predefined solution (the grey dot in the small circle). No efforts are 
made to offer customizations that corresponds to idiosyncratic needs of customers. Thus, the boundary as to what solutions that are offered coincides with the single selectable alternative. This type of solution space is here referred to as a single-point discrete solution space (SPDSS). Each individual product would then be represented as a separate SPDSS. The actual delivery of the product can be based on inventory (maketo-stock, MTS) or made to customer order (make-to-order, MTO) but, in any case only one specific predefined solution is offered. Mass customization approaches have, on the other hand, a solution space constituted by a set of predefined solutions that have been established based on speculation of customers' needs (the larger circle with several discrete dots). It thus differs from SPDSS by predefining multiple solutions and that the specific solution is configured based on customer requirements. Therefore, this type of solution space is referred to as a multiple-point discrete solution space (MPDSS). This case is in line with the assumptions of the product delivery strategy assemble-to-order (ATO) or more specifically CTO. The complete set of predefined solutions thus result in a de facto actual boundary of the solutions that can be offered. Postdefined solutions can be associated with customizations that are individualized. The definition of the solution is here driven by commitment, giving customers more freedom when customizing at the expense of longer delivery lead time compared to predefined solutions. The boundary of the customer's freedom is less distinct than for mass customization and standardization (i.e. where standardization basically represent mass production). It can drive the supplier to occasionally go outside their comfort zone, take on challenging commitments and in that way expand their solution space, which is represented by a potential boundary. This type of solution space is referred to as a continuous solution space (CSS) and is usually associated with ETO.

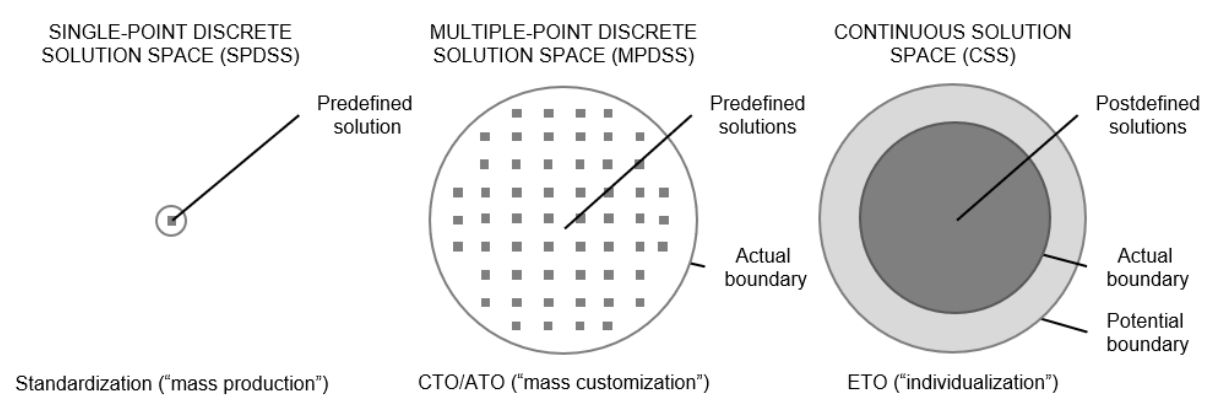

Fig. 1. Categories of solution spaces

As a SPDSS does not offer any customization possibilities, it is not included below. Focus is directed towards MPDSS and CSS and how they require different measures to be developed. The intention of developing the solution space for a case of MPDSS is to further delineate what customizations the supplier can offer. To attain a refined MPDSS, the supplier must have a good understanding of the product attributes of which customers' needs diverge [4]. Suppliers with a CSS are on the other hand devoted to developing competence to understand customers' needs and competence to engineer products fulfilling those needs[5]. 


\section{$5 \quad$ Solution spaces and customization}

The different solution spaces, MPDSS and CSS, can be further delineated based on being driven by speculation or by commitment as in Table 1. A MPDSS requires rules that are established on speculation but used after the CODP. A CSS on the other hand requires the competences of an engineer, designer or alike. These competences are established based on speculation (SD) but are in fact used based on commitment (CD).

Table 1. Solution spaces and flow drivers

\begin{tabular}{lll}
\hline & MPDSS & CSS \\
\hline Speculation driven (SD) & Establish rules & Establish competences \\
Commitment driven (CD) & Use rules & Use competences \\
\hline
\end{tabular}

The full potential of MPDSS can only be reached if customized solutions can be provided while maintaining stability in the production process. This would enable customizations to be provided repeatedly while maintaining a cost-efficient system allowing the customization to be provided at relatively low cost. Postdefined solutions (CSS) are generally intended for less cost sensitive customer segments that require a greater freedom of choice. It is a common assumption in the literature that postdefined solutions (CSS) are delivered only once. However, in practice postdefined solutions can be delivered more than once and this is usually not covered in the literature. In addition to $\mathrm{CD} / \mathrm{SD}$ for solution spaces, it is therefore also important to consider if there is one delivery or multiple separated deliveries for each solution. Combining single delivery and multiple deliveries with the three categories of solution spaces as in Table 2, it is possible to identify five solution/delivery scenarios.

Table 2. Solution spaces and delivery scenarios

\begin{tabular}{llll}
\hline & SPDSS & MPDSS & CSS \\
\hline Single delivery (S) & N/A & CTO/S & ETO/S \\
Multiple deliveries (M) & MTS (MTO) & CTO/M & ETO/M \\
\hline
\end{tabular}

A predefined solution from SPDSS (mass production) of which there is only one delivery is not applicable, as the risk would be too high to establish customization rules on speculation for only one future delivery. Instead, the actual solution must be delivered repeatedly, making it a standardized mass production MTS scenario (possibly MTO if the volumes are low). A solution generated from a MPDSS implies a CTO-production approach (sometimes also referred to as ATO). Such a solution sometimes has the potential to be delivered for multiple commitments and accordingly, the production approach can be divided into a single (CTO/S) or multiple delivery scenario (CTO/M). The same goes for CSS, where postdefined solutions can be delivered for both single $(\mathrm{ETO} / \mathrm{S})$ and multiple (ETO/M) deliveries. 


\section{Conclusions}

To conclude, this research has identified different customization scenarios and accounted for flow drivers, solution spaces, delivery scenarios and product delivery strategies. Furthermore, it has introduced the notion of postdefined solutions which corresponds to individualizations and stand in contrast to predefined solutions and mass customization. The research has also identified three categories of solution spaces that refer to the set of possible solutions for customization.

Three managerial implications have been identified. First, the notion of postdefined solutions can give insights into the role of engineers in customization settings. Second, the categories of solution spaces can contribute to the understanding of both operational and strategic implications that customizations have on supplying suppliers. Third and finally, the research addresses the importance of considering delivery scenarios for both types of solution spaces. This has to a noticeable extent been overlooked in previous customization research.

The research provides a foundation for various directions of further research. The categories of solution spaces could be further refined to also cater for hybrid solutions where a combination of predefined and postdefined solutions are offered. A second avenue is to actually extend the delivery scenario aspect to not only cover multiple deliveries for a single proposal but also involve the potential of multiple proposals supporting a higher level of repetitiveness and its implications for improvement and learning.

\section{Acknowledgements}

This research was performed in collaboration with industry in the project The Whispering Game (KKS-20160210), funded by the research environment SPARK at Jönköping University and the Knowledge foundation.

\section{References}

1. Hoekstra, S., Romme, J.: Integrated Logistical Structures. In. McGraw-Hill, London, (1992)

2. Wacker, J.G.: A definition of theory: research guidelines for different theory-building research methods in operations management. Journal of operations management 16(4), 361-385 (1998).

3. Piller, F.T.: Mass customization: reflections on the state of the concept. International journal of flexible manufacturing systems 16(4), 313-334 (2004).

4. Salvador, F., De Holan, P.M., Piller, F.T.: Cracking the code of mass customization. MIT Sloan management review 50(3), 71 (2009).

5. Engström, A., Wikner, J.: Identifying Scenarios for Ambidextrous Learning in a Decoupling Thinking Context. In: IFIP International Conference on Advances in Production Management Systems 2017, pp. 320-327. Springer 